\title{
Rheology of Gastric Mucin Exhibits a pH-Dependent Sol-Gel Transition
}

\author{
Jonathan P. Celli, ${ }^{\dagger}$ Bradley S. Turner, ${ }^{\dagger, \ddagger}$ Nezam H. Afdhal, ${ }^{\ddagger}$ Randy H. Ewoldt, ${ }^{\S}$ \\ Gareth H. McKinley,§ Rama Bansil, ${ }^{*}, \dagger$ and Shyamsunder Erramilli*, ${ }^{*}$ \\ Department of Physics, Boston University, Boston, Massachusetts 02215, Division of Gastroenterology, \\ Beth Israel Deaconess Medical Center and Harvard Medical School, Boston, Massachusetts 02215, and \\ Hatsopoulos Microfluids Laboratory, Department of Mechanical Engineering, Massachusetts Institute of \\ Technology, Cambridge, Massachusetts 02139
}

Received October 7, 2006; Revised Manuscript Received February 4, 2007

\begin{abstract}
Gastric mucin, a high molecular weight glycoprotein, is responsible for providing the gel-forming properties and protective function of the gastric mucus layer. Bulk rheology measurements in the linear viscoelastic regime show that gastric mucin undergoes a $\mathrm{pH}$-dependent sol-gel transition from a viscoelastic solution at neutral $\mathrm{pH}$ to a soft viscoelastic gel in acidic conditions, with the transition occurring near $\mathrm{pH} 4$. In addition to $\mathrm{pH}$-dependent gelation behavior in this system, further rheological studies under nonlinear deformations reveal shear thinning and an apparent yield stress in this material which are also highly influenced by $\mathrm{pH}$.
\end{abstract}

\section{Introduction}

Throughout the body, luminal epithelial surfaces are covered by a secreted layer of mucus consisting predominantly of water (95\%), high molecular weight polymeric glycoproteins known as mucins $(3 \%)$, and other small molecules $(2 \%){ }^{1}$ This layer serves as a lubricant and as a protective barrier shielding epithelial cells from damaging substances. It is perhaps in the stomach that the mucus layer faces the harshest challenges, not only from ingested materials, but also from the highly acidic gastric juice secreted by the stomach itself. During active digestion, the gastric lumen is typically $1 \leq \mathrm{pH} \leq 2$, while the epithelial surface is near neutral $\mathrm{pH}$, and this gradient is maintained across the mucus layer. ${ }^{1-4}$

The mechanical properties of gastric mucus, which are crucial to its protective function and highly relevant to the problem of drug delivery through the mucus layer, are primarily derived from the mucin glycoproteins that form the gel matrix of the mucus. ${ }^{5}$ Mucins consist of a protein core, with domains rich in cysteine or serine, threonine, and proline, with polysaccharide side chains that account for $80 \%$ of the molecular weight. These glycoproteins form large polymeric aggregates up to approximately $20 \mathrm{MDa}$ via disulfide linkages. ${ }^{6}$ Previous studies of the structure and dynamics have led to a model for $\mathrm{pH}$-induced gelation of gastric mucin. Specifically, experiments using atomic force microscopy ${ }^{7}$ (AFM), dynamic light scattering 8,9 (DLS), and hydrophobic dye binding ${ }^{9}$ have demonstrated that $\mathrm{pH}$ plays a vital role in the mucin-mucin interactions that lead to aggregation and gelation. A conformational change from a random coil conformation at neutral $\mathrm{pH}$ to a rodlike conformation at low $\mathrm{pH}$ probably involves the breakage of salt bridges, and this breakage facilitates associations between the hitherto sequestered hydrophobic regions of the protein core. ${ }^{6}$ Electrostatic interactions between the polysac-

\footnotetext{
* To whom correspondence should be addressed. E-mail: shyam@bu.edu (S.E.); rb@bu.edu (R.B.).

$\dagger$ Boston University.

$\$$ Beth Israel Deaconess Medical Center and Harvard Medical School.

$\S$ Massachusetts Institute of Technology.
}

charide side chains of mucin are responsible for maintaining the extended rodlike conformation of $\operatorname{mucin}^{7}$ and play an important role in determining the structure and viscoelastic properties of the gel.

Because of the physiological importance of the mechanical properties of mucus, there have been numerous rheological studies not only on mucus and mucin from the GI tract ${ }^{8,10-19}$ but also on tracheal ${ }^{20-22}$ and cervical ${ }^{23,24}$ mucus. In addition, recent studies have examined the microrheology ${ }^{17-19,25}$ of mucins and mucus. Microrheology studies are particularly relevant to the transport of drugs and infectious agents such as bacteria and viruses across the mucus barrier. However, characterization of the macroscopic bulk rheology is important to physiologically relevant problems such as mucus shedding and the response of the mucus layer to large deformations during peristalsis. The bulk properties are also crucial in medical applications involving the mechanical motion of invasive devices such as endoscopes. ${ }^{26}$

In the case of gastric mucin, bulk rheological measurements also provide a direct probe to investigate the $\mathrm{pH}$-induced solgel transition which may play a vital role in the physiological mechanism by which the stomach is protected from digesting itself. However, to the best of our knowledge, detailed bulk rheology experiments to study the impact of $\mathrm{pH}$ on the mechanical properties of gastric mucin have not been carried out. Previous work using falling ball viscometry ${ }^{8}$ has shown that there is a profound increase in the apparent viscosity at low $\mathrm{pH}$, though these experiments were not able to probe different shear rates and measure the full frequency-dependent linear viscoelastic moduli. Another study, in which the viscosity of partially purified PGM purchased from Sigma Chemicals with added trefoil peptides was examined, did note an increase in viscosity at low $\mathrm{pH}$, although $\mathrm{pH}$ dependence in purified mucin was not studied. ${ }^{16}$ An earlier bulk rheology study in which mucin viscosity at different $\mathrm{pH}$ values was examine $\mathrm{d}^{27}$ did not observe significant variation in the material properties, most likely due to high salt concentrations in the buffer solution, a factor which has been observed (and is further studied in this work) to inhibit the $\mathrm{pH}$-dependent change in rheology. ${ }^{8}$ 


\section{B Celli et al.}

Recently we reported microrheology measurements using a microscope-based DLS instrument to examine the local mechanical properties of a mucin gel at different $\mathrm{pH}$ values by examining the diffusive motion of tracer particles. ${ }^{17}$ These onepoint tracer probe microrheology measurements sample localized microenvironments on submicrometer length scales that are not necessarily expected to agree with the bulk properties of the material. ${ }^{28}$

In this work, as in previous studies, a model mucus system of purified porcine gastric mucin (PGM) is used. Mucus reconstituted by hydration of purified PGM has been shown to duplicate the rheological properties of native mucosal scrapings from pig stomachs. ${ }^{12}$ The porcine animal model is used due to physiological similarities between human and pig stomachs. Moreover, the human MUC5AC gene, which (along with MUC6) is expressed by the gastric mucosa, is homologous to the porcine gastric mucin gene. ${ }^{29}$ Since MUC5AC is expressed in the airway mucosa as well as the GI tract, the results of these studies may also be relevant to airway mucus, the viscoelastic properties of which are a contributing factor in obstructive lung diseases such as cystic fibrosis $(\mathrm{CF})$ and chronic obstructive pulmonary disease.

\section{Materials and Methods}

Materials. Porcine gastric mucin used in these experiments was purified from the mucus scrapings of fresh pig stomachs. The use of purified PGM rather than the commercially available partially purified PGM (Sigma-Aldrich, St. Louis, MO) results in a far more accurate model system as the protease treatment in the preparation of the latter has been shown to degrade the glycoproteins such that the rheological properties are qualitatively different from those of the actual mucus..$^{10,30}$ PGM was purified by Sepharose CL2B chromatography and cesium chloride density gradient ultracentrifugation as described previously. ${ }^{9}$ Lyophilized PGM was prepared for rheology experiments by hydration in sterile $\mathrm{H}_{2} \mathrm{O}$ and buffering to the appropriate $\mathrm{pH}$ using a previously calibrated set of phosphate/succinate buffers. The $\mathrm{pH}$ of the PGM samples was adjusted by mixing appropriate volumes of $100 \mathrm{mM} \mathrm{H}_{3-}$ $\mathrm{PO}_{4} /$ succinic acid and $\mathrm{Na}_{3} \mathrm{PO}_{4} /$ sodium succinate to obtain the indicated $\mathrm{pH}$. The final concentration of $\mathrm{PO}_{4} /$ succinate in the samples was 10 $\mathrm{mM}$. In certain tests noted below, a modified set of buffers prepared to isoionic conditions by addition of $\mathrm{NaCl}$ to a $50 \mathrm{mM} \mathrm{NaCl}$ conductivity standard was used. Buffered PGM solutions were allowed to hydrate and equilibrate for $48 \mathrm{~h}$ before testing.

Reduced mucin was prepared by reacting purified PGM overnight with $10 \mathrm{mM}$ dithiothreitol (DTT) in $10 \mathrm{mM}$ Tris - $\mathrm{HCl}, 0.1 \mathrm{mM}$ EDTA (disodium ethylenediaminetetraacetate), $\mathrm{pH}$ 8.0, and subsequent alkylation with $50 \mathrm{mM}$ iodoacetamide (IAM) in the dark at room temperature. The reduced PGM was dialyzed extensively against deionized water using a 1000 molecular weight cutoff (MWCO) dialysis membrane to remove remaining DTT, IAM, and buffers while retaining any small peptide fragments. Finally, the dialyzed reduced PGM was lyophilized before rehydration and buffering to the desired concentration and $\mathrm{pH}$ as above.

Rheology. TA instruments AR-G2 and AR-2000 stress-controlled rheometers (TA Instruments, New Castle, DE) were used to study the mechanical response of PGM samples in steady shear, oscillatory shear, and step stress modes. In these studies a $40 \mathrm{~mm}$ parallel plate geometry with a solvent trap was used. For most samples, a gap of $250 \mu \mathrm{m}$ was sufficient to distribute the sample evenly over the entire surface area of the plate. The rheometer is equipped with a Peltier plate to regulate the sample temperature to $22.0^{\circ} \mathrm{C}$. In all cases, an initial stress sweep with an oscillatory shear stress of increasing amplitude at a constant frequency of $\omega=0.5 \mathrm{rad} / \mathrm{s}$ was performed to determine the region of linear response for a given sample. On the basis of this test, a value of applied stress within the linear regime was then used in the subsequent
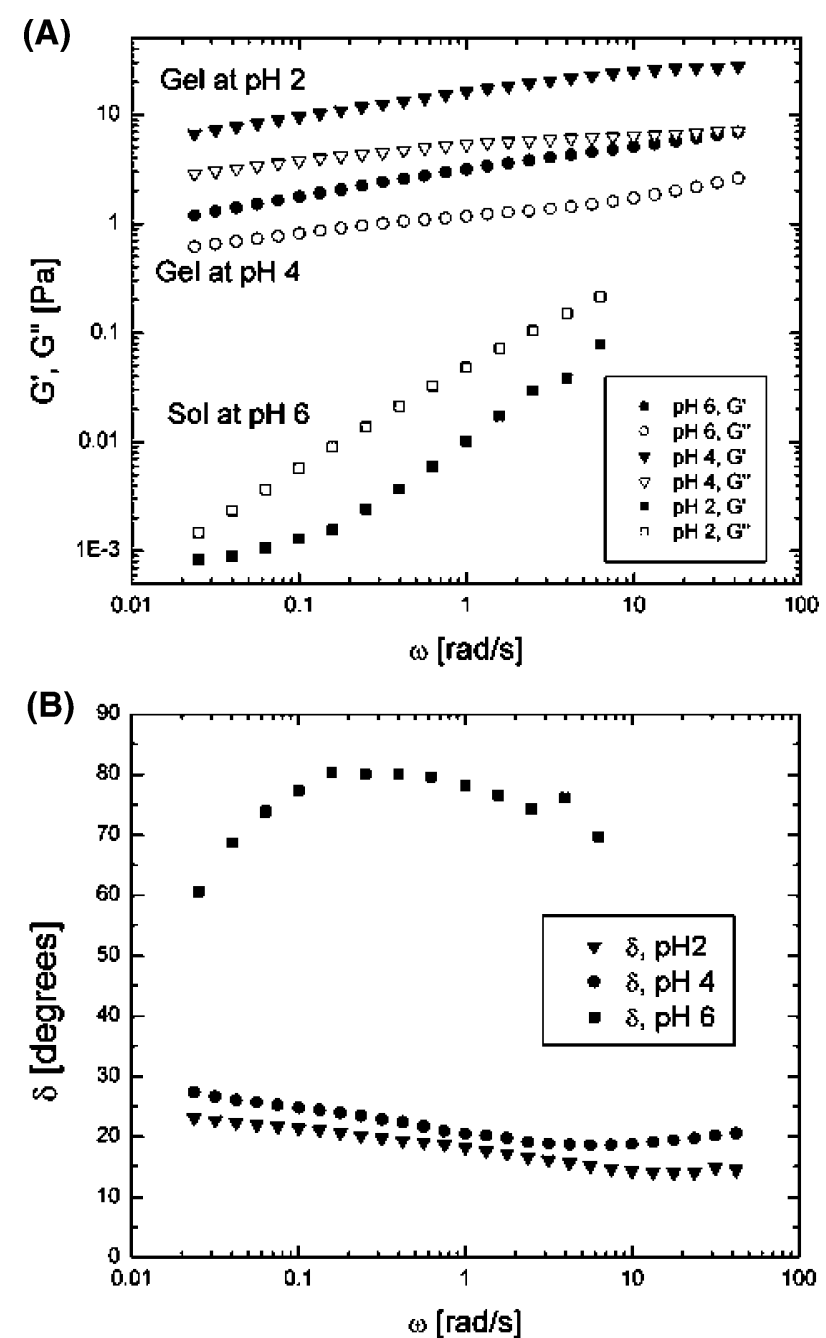

Figure 1. (A) Linear viscoelastic moduli, $G^{\prime}(\omega)$ and $G^{\prime \prime}(\omega)$, for 15 $\mathrm{mg} / \mathrm{mL}$ PGM at pH $2(\boldsymbol{\nabla}, \nabla), \mathrm{pH} 4(\bullet, O)$, and pH $6(\boldsymbol{\square}, \square)$. (B) Phase angle, $\delta$, as a function of frequency for the same data. The PGM samples at $\mathrm{pH} 2$ and 4 exhibit the characteristic response of a gel, with $G^{\prime}(\omega)$ dominant over $G^{\prime}(\omega)\left(\delta<45^{\circ}\right)$ for all frequencies probed. In contrast, PGM is clearly a solution at $\mathrm{pH} 6$, with $G^{\prime \prime}(\omega)$ greater than $G^{\prime}(\omega)\left(\delta>45^{\circ}\right)$ for all frequencies accessed.

creep test and frequency sweeps on the sample. In addition to these three tests, a "flow test" (a series of step increases in the steady-state stress) was also carried out on most samples to obtain the steady shear viscosity as a function of shear stress.

\section{Results and Discussion}

PH-Dependent Sol-Gel Transition. Frequency sweeps in

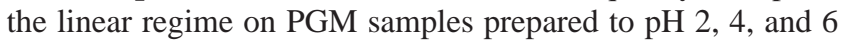
shown in Figure 1 present clear evidence of a dramatic change in viscoelastic behavior at low $\mathrm{pH}$. At $\mathrm{pH} 2$ and 4 the storage modulus, $G^{\prime}$, is dominant over the loss modulus, $G^{\prime \prime}$, for all frequencies probed, indicating a gel-like material response. At $\mathrm{pH} 6$ the values of both components of the complex modulus are several orders of magnitude lower than in the low-pH (gelled) samples, and the loss modulus, $G^{\prime \prime}$, is dominant over the storage modulus, $G^{\prime}$, indicative of a liquidlike response. Another way of quantifying the gel strength and gelation is to look at the phase angle, $\delta$, given by the relation $\tan \delta=G^{\prime \prime} / G^{\prime}$. The lower the value of $\delta$, the more elastic the material, where a value of $\delta \leq 45^{\circ}$ is generally indicative of a sample that has gelled while a value of $\delta \geq 45^{\circ}$ corresponds to a sample in the 


\section{Biomacromolecules}

sol state. On the basis of this criterion, it is clear that at $\mathrm{pH} 6$ PGM is in the solution state while at $\mathrm{pH} 2$ it is slightly more strongly gelled than at $\mathrm{pH} 4$. Because the $\mathrm{pH} 6$ sample has extremely small values of both viscoelastic moduli, the data were truncated due to the onset of inertial effects in the instrument at higher frequencies.

We also noted that, in the absence of a pH buffer, PGM hydrated in deionized water drives the $\mathrm{pH}$ to 4.05 , and the sample is in fact more strongly gelled than in the case of $\mathrm{pH} 2$ PGM. The high strength of the gel in the absence of buffer is likely due to the effect of partial charge screening by ions in the buffer, as discussed below. It should be noted that other rheological studies on reconstituted purified $\mathrm{PGM}^{12,14,15}$ in which no $\mathrm{pH}$ buffer was used in the final hydration of the lyophilized PGM, but in which the sample is observed to form a gel, are consistent with data presented here because, in the absence of added buffer, these were probably effectively pH 4 PGM gels. The values reported for low-pH gel samples here are approximately consistent given differences in glycoprotein concentration with previously reported values of the frequencydependent moduli of native gastric mucus. ${ }^{12}$ The same appears to be true of native mucus secretions from the trachea, ${ }^{20}$ while cervical mucus ${ }^{24}$ has lower values of both moduli.

Further evidence of the $\mathrm{pH}$-dependent sol-gel transition is apparent from creep test data, plotted in Figure 2. At pH 2 there is strong evidence of elastic recovery in the sample, while at $\mathrm{pH} 6$, the sample essentially flows and no elastic recovery could be reliably measured. A measure of the steady shear viscosity can also be obtained from the long-time response of the creep compliance, $J(t)$. For a linear viscoelastic material at times $t \approx$ $\lambda$ (the relaxation time), we expect

$$
J(t) \equiv \frac{\gamma(t)}{\sigma_{0}}=a+\frac{t}{\eta_{0}}
$$

where $\gamma(t)$ is the time-dependent strain response, $\sigma_{0}$ is the applied step stress, $\eta_{0}$ is the viscosity at that specific stress or shear rate, and $a$ is an offset. Fitting a straight line to the $\mathrm{pH} 2$ compliance data at late times, we obtain $\mathrm{d} J / \mathrm{d} t=\eta_{0}{ }^{-1}=0.00022$ $(\mathrm{Pa} \cdot \mathrm{s})^{-1}$, and solving for $\eta_{0}$ yields a value of $4500 \mathrm{~Pa} \cdot \mathrm{s}$. Repeating this analysis for the $\mathrm{pH} 6$ data yields a viscosity of $0.20 \mathrm{~Pa} \cdot \mathrm{s}, 4$ orders of magnitude lower than in the $\mathrm{pH} 2$ sample. At $\mathrm{pH} 4$ the creep compliance is qualitatively the same as the compliance at $\mathrm{pH} 2$, and applying the same analysis to the $\mathrm{pH}$ 4 data, the calculated value of $\eta_{0}$ is $160 \mathrm{~Pa} \cdot \mathrm{s}$.

Ionic Strength at Constant pH. It has been previously reported that the presence of salts impacts mucin rheology. $8,31,32$ The data presented in Figure 3 demonstrate that, as the salt concentration is increased at $\mathrm{pH}$ 2, PGM remains a gel up to a $\mathrm{NaCl}$ concentration of $100 \mathrm{mM}$, but the sample at $200 \mathrm{mM}$ becomes a solution. At these very high salt concentrations PGM is a solution regardless of $\mathrm{pH}$; however, even the $200 \mathrm{mM}$ sample does cross over to a more solidlike response at high frequencies as is typical for a polymeric liquid. ${ }^{33}$ The 100-fold loss of elasticity of mucin gels with increasing salt seen here is opposite the behavior of covalently cross-linked polyelectrolytic gels whose elastic modulus increases with increasing salt concentration, ${ }^{34}$ suggesting that, in the case of mucin, which is a physical gel, the screening of electrostatic interactions decreases the degree of cross-linking and entanglement of the physical gel network. The decrease of effective charge reduces the electrostatic repulsion of the sugar side chains, which adopt a less extended conformation. Charge screening would also impact the stability of salt bridges in the protein core and affect
Rheology of Gastric Mucin
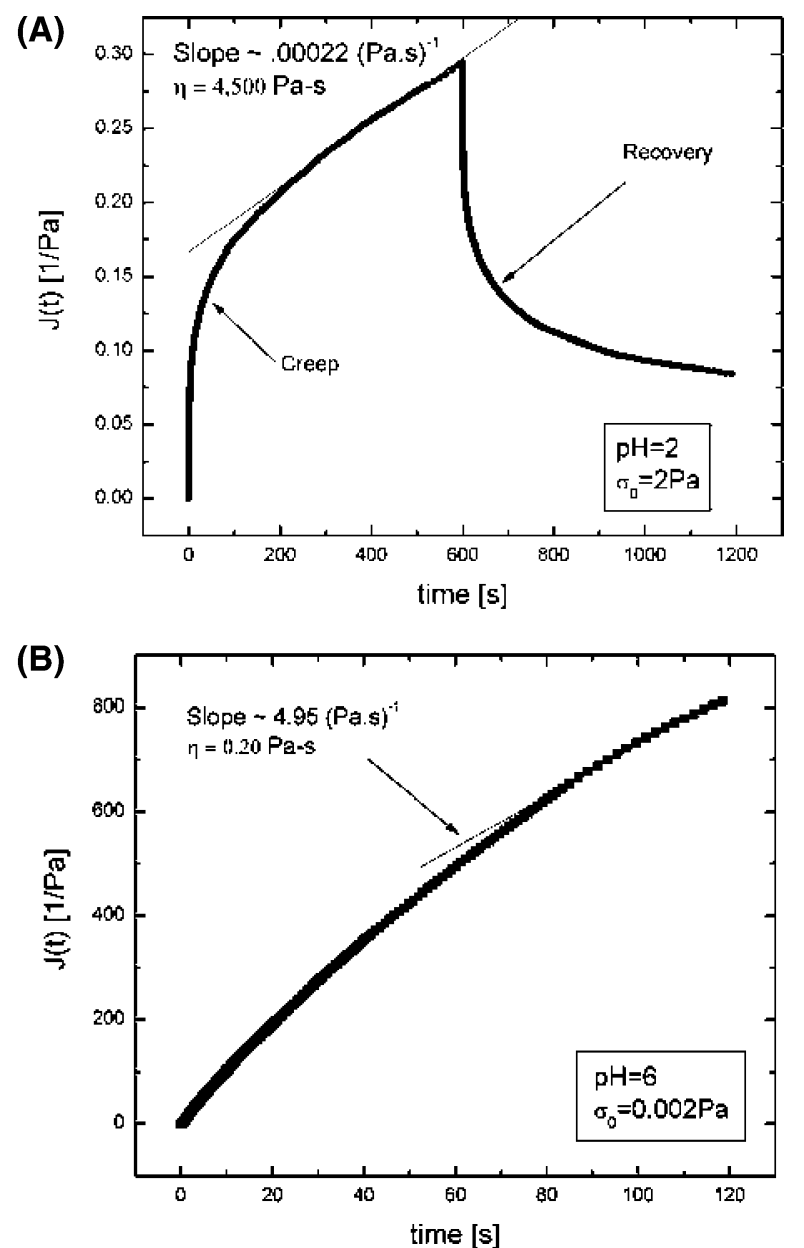

Figure 2. Creep compliance, $J(t)=\gamma(t) / \sigma_{0}$, versus time for PGM at $\mathrm{pH} 2$ (A) and $\mathrm{pH} 6$ (B). Both tests were conducted at a value of applied stress, $\sigma_{0}$, within the linear regime for that sample. For the $\mathrm{pH} 2$ sample, $\sigma_{0}=2 \mathrm{~Pa}$, while at $\mathrm{pH} 6, \sigma_{0}=0.002 \mathrm{~Pa}$. There is an obvious qualitative contrast between the two samples in that there is evidence of significant elasticity and very little irreversible creep at $\mathrm{pH} 2$. Because of the lack of elasticity in the $\mathrm{pH} 6$ sample, recovery could not be reliably measured. From linear fits to the compliance at late times, the viscosity, $\eta_{0}$, at $\mathrm{pH} 2$ and 6 is found to be 4500 and 0.20 $\mathrm{Pa} \cdot \mathrm{s}$, respectively.

the folding of the protein and influence its ability to form intermolecular associations required for gelation. At the other extreme, the gel in pure deionized water has strong polyelectrolytic interactions and is highly swollen.

These studies of the effect of salt concentration on mucus rheology may be relevant to airway mucus, which also contains the secreted gel-forming mucin MUC5AC. ${ }^{35}$ The observation of a decrease in mucin gel strength is consistent with recent studies which have shown that inhalation of hypertonic saline aids in mucus clearance for cystic fibrosis patients. ${ }^{36}$

Observation of the Nearly Critical Gel at pH 4. To ensure that the rheological changes in the gel properties presented above were entirely driven by $\mathrm{pH}$ rather than ionic strength, which does vary slightly with the $\mathrm{pH}$ of the buffer solution, rheology experiments at varying $\mathrm{pH}$ were repeated on samples of identical ionic strength (Figure 4). Isoionic buffers were prepared by measuring the conductivities of the $\mathrm{PO}_{4} /$ succinate buffer at the indicated $\mathrm{pH}$ and then adding $\mathrm{NaCl}$ to a final ionic strength equivalent to $50 \mathrm{mM} \mathrm{NaCl}$. As expected, the small quantities of additional salt added here did not change qualitatively the stiffening of the material at low $\mathrm{pH}$. However, under these conditions, it is observed that PGM at $\mathrm{pH} 4$ is very nearly a 


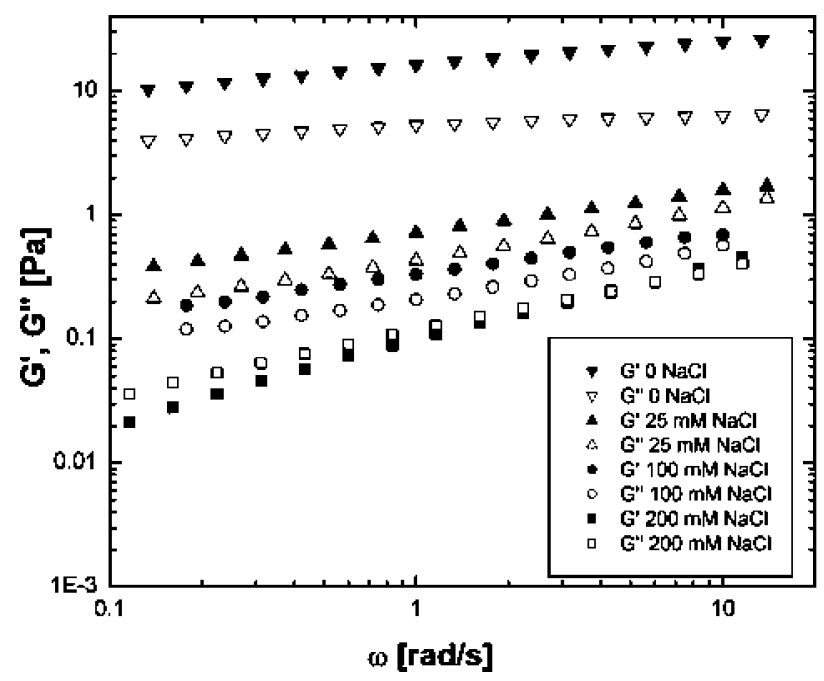

Figure 3. $G^{\prime}(\omega)$ and $G^{\prime \prime}(\omega)$ for PGM at pH 2 with no added $\mathrm{NaCl}(\boldsymbol{\nabla}$, $\nabla)$, with $25 \mathrm{mM} \mathrm{NaCl}(\mathbf{\Delta}, \triangle)$, with $100 \mathrm{mM} \mathrm{NaCl}(\bullet, \bigcirc)$, and with 200 $\mathrm{mM} \mathrm{NaCl}(\boldsymbol{\square}, \square)$. At highly elevated salt concentrations PGM no longer gels regardless of $\mathrm{pH}$ but still exhibits an elastic dominant response at higher frequencies.

critical gel by the criteria of Chambon and Winter, that $G^{\prime \prime}=$ $G^{\prime} \tan \delta=S \omega^{n} \Gamma(1-n) \cos \delta$, where $S$ is the gel stiffness parameter and the scaling exponent $n$ is related to the phase angle by $\delta=n \pi / 2 \cdot{ }^{37,38}$ For these data, the average value of the phase angle is $0.61 \pm 0.04 \mathrm{rad}$, indicating a value of the scaling exponent, $n$, of $0.39 \pm 0.02$. Power law fits of the form $G^{\prime}(\omega)$ $\approx G^{\prime \prime}(\omega) \approx \omega^{n}$ agreed well with the experimental data and yielded values of $n=0.38 \pm 0.002$ and $n=0.41 \pm 0.006$ for $G^{\prime}(\omega)$ and $G^{\prime \prime}(\omega)$ fits, respectively, each within $5 \%$ of the value independently calculated from the phase angle relation, $n=0.39$ \pm 0.02 . Percolation theory calculations presented by Muthukumar $^{39,40}$ suggest that the scaling exponent, $n$, is related to a fractal dimension, $d_{\mathrm{f}}$, by

$$
n=\frac{d\left(d+2-2 d_{\mathrm{f}}\right)}{2\left(d+2-d_{\mathrm{f}}\right)}
$$

where $\mathrm{d}$ is the spatial dimension (and is equal to 3 here) and $d_{\mathrm{f}}$ relates the molecular weight of the polymeric aggregate, $M$, to its spatial size, $R$, by $R_{\mathrm{f}} \approx M$. In this case, solving yields $d_{\mathrm{f}}=$ $2.13 \pm 0.02$, lower than the value of 2.5 predicted for percolation clusters at threshold ${ }^{41}$ and comparable to values observed in $\iota$-Carrageenan gels. ${ }^{42}$ The observation of a nearly critical gel at $\mathrm{pH} 4$ is intriguing in view of recent studies showing that the surface $\mathrm{pH}$ in resting mouse stomachs is regulated to $\mathrm{pH} 4 .{ }^{43}$

Yield Stress. Constant-frequency oscillatory stress sweeps for $\mathrm{pH} 2$ and 6 PGM are plotted in Figure 5. Note that, at stresses beyond the linear regime, the values of $G^{\prime}$ and $G^{\prime \prime}$ are highly approximate since the material response is not a single harmonic sinusoid. PGM gel samples all have a qualitatively similar response, with $G^{\prime}$ dominant over $G^{\prime \prime}$ in the linear regime, indicative of a gel state. As stress is ramped above a critical stress of $\tau_{\mathrm{y}} \approx 10 \mathrm{~Pa}$, the gel catastrophically fails and the rheology is dominated by the viscous loss modulus, with $G^{\prime \prime}$ greater than $G^{\prime}$. This critical stress may be interpreted as an apparent yield stress, since the elastic properties decrease by at least 2 orders of magnitude over a narrow range of stress. ${ }^{44}$ At pH 6 (shown in Figure 5b), the concept of a yield or critical stress is not applicable because even at low stresses the response is dominated by the loss modulus; however, both components of the modulus show significant stress softening above an
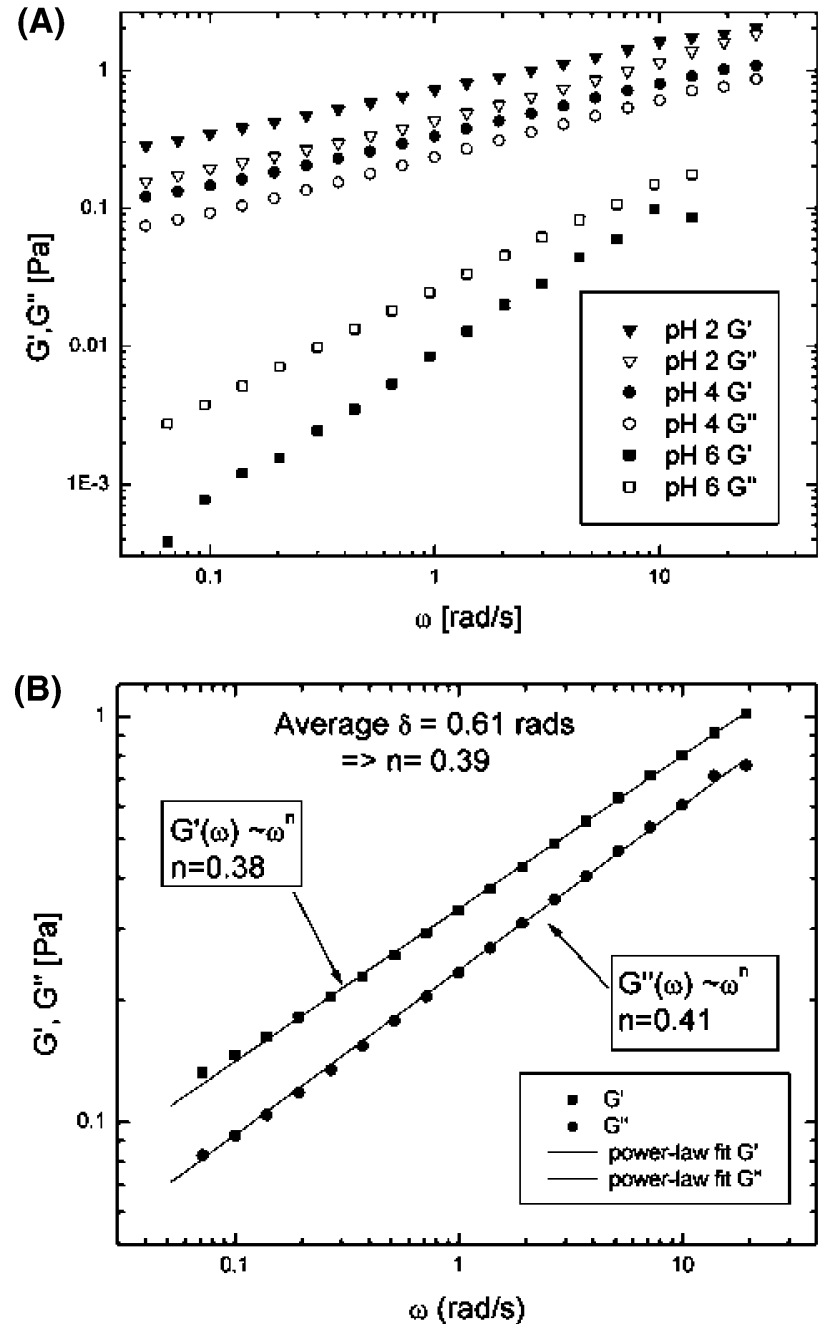

Figure 4. (A) Linear viscoelastic moduli, $G^{\prime}(\omega)$ and $G^{\prime \prime}(\omega)$, for PGM at $\mathrm{pH} 2(\boldsymbol{\nabla}, \nabla), \mathrm{pH} 4(\bullet, \bigcirc)$, and $\mathrm{pH} 6(\boldsymbol{\square}, \square)$, prepared to isoionic conditions as described in the text. (B) Detail of the $\mathrm{pH} 4$ data showing power-law fits of the form $G^{\prime}(\omega) \approx G^{\prime \prime}(\omega) \approx \omega^{n}$. The value of $n$ from each component of the complex modulus is within $5 \%$ agreement with $n=0.39$ from the criteria that $\tan \delta=\tan (n \pi / 2)$ for a critical gel.

applied stress of about 0.1 Pa. In a pH 4 gel, the critical stress 291 behavior is approximately the same as in the $\mathrm{pH} 2$ gel, with $\tau_{\mathrm{y}} 292$ $\approx 10 \mathrm{~Pa}$. Note that the stresses reported here are maximum 293 amplitudes of the applied sinusoidal oscillatory stress, so the 294 rms value of the yield stress is actually $\tau_{\mathrm{y}} / \sqrt{ } 2 \approx(10 \mathrm{~Pa}) / \sqrt{ } 2=295$ $7.07 \mathrm{~Pa}$. Given the dramatic stress softening by orders of 296 magnitude observed here, the approximate measurements of $G^{\prime} 297$ and $G^{\prime \prime}$ in the nonlinear regime are adequate to characterize 298 this behavior. However, if more accuracy were required in 299 examining the nonlinear viscoelastic properties, further experi- 300 ments to study this could include strain-dependent relaxation 301 modulus measurements as conducted by Xu et al. in studies of 302 the rheology of F-actin networks ${ }^{45}$ or Fourier transform rheology. ${ }^{46}$

An apparent yield stress has been observed in other mucus systems as well. Pedal mucus from terrestrial gastropods (snails and slugs) is known to have a yield stress, and the yield stress has been identified as the primary attribute which allows these creatures to crawl up inclined surfaces such as vertical walls. ${ }^{47}$ Additionally, Taylor et al. have reported data that indicate dramatic stress softening in PGM. ${ }^{14}$ Furthermore, in the same work it is shown that scrapings from different parts of the stomach actually exhibit different rheological properties. In our work, the scrapings were obtained from entire stomachs; 

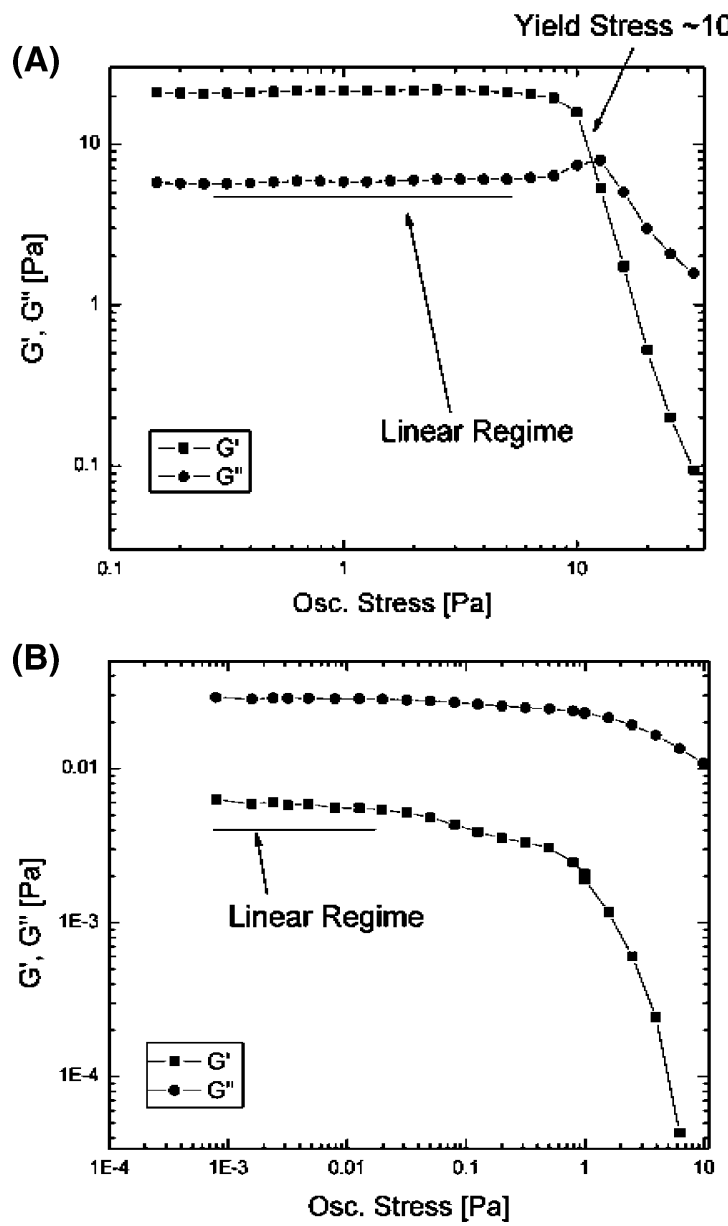

Figure 5. Stress sweep data for (A) pH 2 PGM (gel), $15 \mathrm{mg} / \mathrm{mL}$, and (B) pH 6 PGM (sol), plotted as $G^{\prime}(\mathbf{\square})$ and $G^{\prime \prime}(\bullet)$ versus the amplitude of the applied oscillatory shear stress at a constant frequency of $\omega=0.5 \mathrm{rad} / \mathrm{s}$. In the $\mathrm{pH} 2$ sample, as stress is increased beyond the linear regime, the gel yields and begins to flow just above $10 \mathrm{~Pa}$.

however, the results for yield stress reported here are consistent with an appropriately weighted average of the yield stress values apparent from examination of data presented by Taylor et al. for PGM from two different regions of the stomach. The trends of the two curves here are more like the "shear-resistant" gel described in the work of Taylor et al., in that there is a slight rise in the loss modulus before the dramatic drop in both moduli. This behavior is seen in a variety of materials tested in largeamplitude oscillatory shear (LAOS) and has been identified as "weak strain overshoot" (or type III) behavior by Hyun et al. ${ }^{48}$ These materials share the common property that they form a complex structure that resists against deformation up to a certain strain where $G^{\prime \prime}$ increases, but then fail dramatically at higher strains. Hyun et al. have associated type III behavior with systems that have extended backbones and long side chains that are electrically charged, a description which is very consistent with our knowledge of mucin's extended rodlike conformation, polyelectrolytic properties, and liquid crystalline structure. ${ }^{6,49}$

The value of the yield stress can also be used to calculate an estimate of the maximum thickness of the layer on a vertical surface that can be supported under the influence of gravity by

$$
\tau_{\mathrm{y}}=\rho g h_{\mathrm{c}}
$$

where $\tau_{\mathrm{y}}$ is the yield stress, $\rho$ is the density of the material, $g$ is the acceleration due to gravity, and $h_{\mathrm{c}}$ is the thickness of the

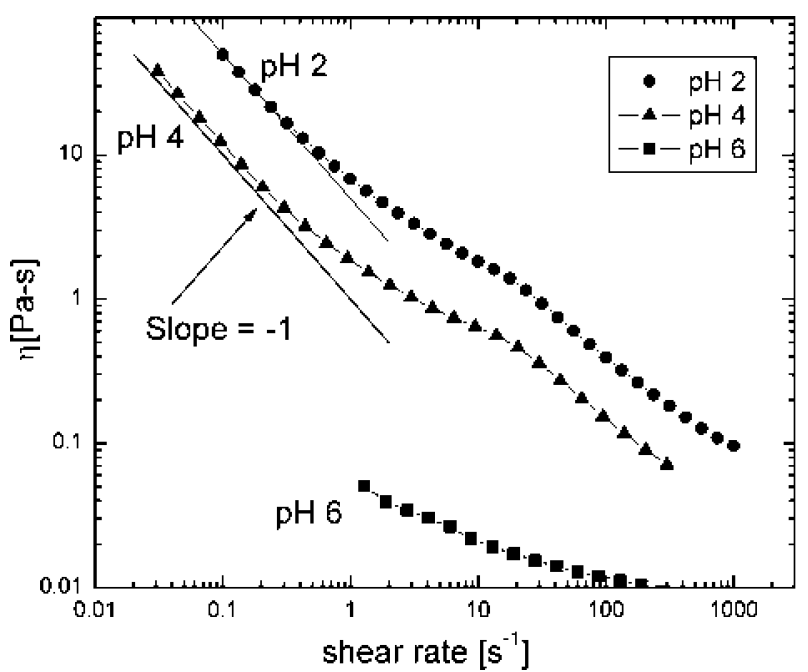

Figure 6. Flow test data for $\mathrm{PGM}, 15 \mathrm{mg} / \mathrm{mL}$, showing steady shear viscosities at $\mathrm{pH} 2(\boldsymbol{\bullet}), \mathrm{pH} 4(\mathbf{\Delta})$, and $\mathrm{pH} 6(\boldsymbol{\square})$. The apparent viscosity (stress/shear rate) is plotted as a function of shear rate, with lines of slope -1 showing agreement with the form $\log \eta=\log \tau_{\mathrm{y}}-\log \bar{\gamma}$ for gel samples as described in the text. The lower $\mathrm{pH}$ samples are dramatically shear thinning, decreasing in viscosity by about 3 orders of magnitude over 4 decades in shear rate and approaching a constant (yield) stress at low shear rates.

mucus layer. In this case, solving for $h_{\mathrm{c}}$, using $\tau_{\mathrm{y}} \approx 10 \mathrm{~Pa}, \rho \approx$ $10^{-3} \mathrm{~kg} / \mathrm{m}^{3}$, and $g=9.8 \mathrm{~m} / \mathrm{s}^{2}$ yields $h_{\mathrm{c}} \approx 1 \mathrm{~mm}$. This upper limit for film thickness is consistent with measurements of mucus layer thickness in vivo $^{3}$ in which the thickest mucus observed in the colon was measured to be $\sim 830 \mu \mathrm{m}$. The approximate agreement of this estimate with actual mucus layer thicknesses suggests that gravity may in fact play a role in limiting the thickness of the mucus layer. The observation of an apparent yield stress in gastric mucin may have further physiological relevance given the large shear stresses that the stomach is subject to during digestion.

Shear Thinning. All PGM samples studied in this work exhibited a highly non-Newtonian shear thinning behavior in steady shear flow tests, as previously observed by other researchers in tests with degraded PGM purchased from Sigma Chemicals. ${ }^{16,49,50}$ In Figure 6, the apparent viscosity (stress divided by shear rate) measured in steady shear flow is plotted as a function of shear rate for PGM buffered to $\mathrm{pH} 2,4$, and 6 . The apparent viscosity at all shear rates probed increases with decreasing $\mathrm{pH}$, and the approach to a constant (yield) stress can be seen in the gel samples ( $\mathrm{pH} 2$ and 4). For the $\mathrm{pH} 2$ sample, this line is close to $\eta=\left(\tau_{\mathrm{y}} / \sqrt{2}\right) / \bar{\gamma}=(7.07 \mathrm{~Pa}) / \bar{\gamma}$ or $\log$ $\eta=\log 7.1-\log \dot{\gamma}$ as expected from the independent measurement of yield stress discussed above, where the second term in the logarithmic form of the expression gives rise to the slope -1 lines shown in Figure 6.

It is worth noting that the viscosity data obtained here in steady shear experiments are self-consistent with the viscosity information obtained from the creep test data reported above. In comparing viscosity values obtained from a steady shear test with those from a creep test, it is important to determine the relevant shear rate in the latter test. For the $\mathrm{pH} 2$ sample, $\bar{\gamma}_{\text {creep }}$ $=\sigma_{0}(\mathrm{~d} J(t) / \mathrm{d} t)=2 \mathrm{~Pa} \times 0.00022(\mathrm{~Pa} \cdot \mathrm{s})^{-1}=4.4 \times 10^{-4} \mathrm{~s}^{-1}$, in which case the true steady-state shear viscosity for the $\mathrm{pH} 2$ gel, combining the yield stress information with the creep tests data, would be $\eta(2 \mathrm{~Pa})=7.07 /\left(4.4 \times 10^{-4}\right)=15900 \mathrm{~Pa} \cdot \mathrm{s}$. In Figure 6 these data points would be up and to the left and would lie close to the trend line shown. Shear thinning behavior, 


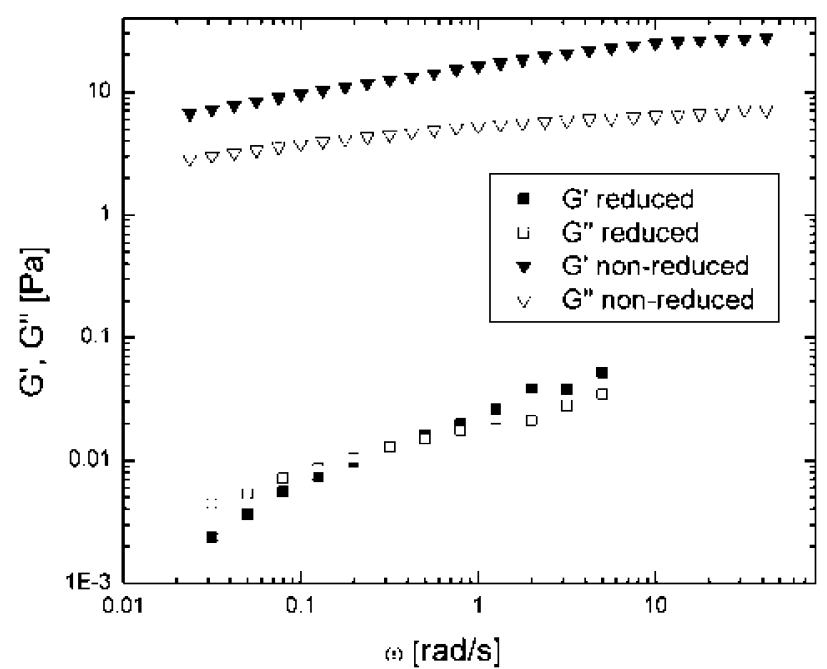

Figure 7. Linear viscoelastic moduli, $G^{\prime}(\omega)$ and $G^{\prime \prime}(\omega)$, for PGM at $\mathrm{pH} 2(\mathbf{\nabla}, \nabla)$ and DTT-reduced PGM at pH $2(\mathbf{\square}, \square)$, showing the dramatic change from elastic gel to viscoelastic solution after reduction of PGM into subunits.

combined with the $\mathrm{pH}$-dependent yield stress behavior discussed above, may perhaps play a role in the mechanism of mucus shedding.

Reduced Mucin. To further examine the nature of the mucin-mucin interactions that give rise to gelation, samples of gastric mucin reduced into their monomer subunits by DTT and IAM were also prepared and studied. Frequency sweep data for $15 \mathrm{mg} / \mathrm{mL}$ DTT reduced PGM at $\mathrm{pH} 2$ (Figure 7) shows that even at low $\mathrm{pH}$ the reduced PGM exhibits the material response of a viscoelastic solution rather than a gel. This observation that reduced PGM does not gel at low $\mathrm{pH}$ is also consistent with previous $\mathrm{AFM}^{51}$ and $\mathrm{DLS}^{8}$ studies on reduced mucin. At $\mathrm{pH} 6$ the reduced PGM displays essentially no measurable elasticity at the concentration studied and is only approximately twice as viscous as water $(1 \mathrm{mPa} \cdot \mathrm{s})$, so even at very low stresses it was not possible to measure the complex modulus. It is however worth noting that even when reduced into subunits, the rheology of PGM still exhibits some $\mathrm{pH}$ dependence, indicating that $\mathrm{pH}$-dependent interactions still exist and impart some fluid viscoelasticity to the bulk sample, but because of the reduced chain length, a macromolecular network is not formed.

\section{Conclusions}

These experiments show conclusively through bulk mechanical observations that PGM undergoes a $\mathrm{pH}$-dependent solgel transition. As noted in previous work, ${ }^{6-9,52}$ a $\mathrm{pH}$-dependent sol-gel transition of gastric mucin may be crucial to the mechanisms that protect the epithelium from secreted acid in mammalian stomachs. Furthermore, given that the mucus layer exists across a dramatic $\mathrm{pH}$ gradient from the lumen to the epithelial surface, an understanding of the effect of $\mathrm{pH}$ on the material properties of the mucus gel is an important part of gaining a complete understanding of the rheology of this system. Furthermore, an understanding of the bulk mechanical properties of mucus at varying $\mathrm{pH}$ may be relevant to the response of the entire mucus layer to large shear stresses during digestion and the shedding of the layer which occurs daily. They may also be relevant to the design of invasive mechanical devices such as endoscopes. ${ }^{26}$

The data presented here are consistent with the observation of a pH-dependent conformational change in PGM that has been observed in $\mathrm{AFM}^{7}$ and DLS ${ }^{8,9}$ experiments on dilute solutions 417 and are also consistent with other recent investigations on the 418 diffusive motion of submicrometer particles embedded in 419 PGM. ${ }^{17}$ Additional experiments have shown that gelation and 420 gel strength are influenced not only by $\mathrm{pH}$, but also by variations 421 in ionic strength. The current model for PGM gelation involves 422 the breakage of salt bridges at low $\mathrm{pH}$, exposing hydrophobic 423 domains on the protein core, which then form junctions. In the context of this model, the data presented here provide some insight into the relative contributions of these hydrophobic interactions on the protein core and the electrostatic interactions of the polysaccharide side chains.

Acknowledgment. We gratefully acknowledge support from the National Science Foundation and the National Institutes of Health. R.H.E. acknowledges the National Science Foundation Graduate Research Fellowship Program.

Supporting Information Available. Viscoelastic moduli for PGM hydrated only in deionized water with no $\mathrm{pH}$ buffer and salt concentrations for the isoionic buffer preparation used in the relevant section of this paper. This material is available free of charge via the Internet at http://pubs.acs.org.

\section{References and Notes}

(1) Allen, A. Structure and function of gastrointestinal mucus. In Physiology of the gastroenterology tract, 1st ed.; Johnson, L., Ed.; Raven Press: New York, 1981; pp 617-39.

(2) Allen, A.; Leonard, A. J.; Sellers, L. A. J. Clin. Gastroenterol. 1988, 10 Suppl 1, S93-8.

(3) Atuma, C.; Strugala, V.; Allen, A.; Holm, L. Am. J. Physiol.: Gastrointest. Liver Physiol. 2001, 280 (5), G922-9.

(4) Allen, A.; Flemstrom, G. Am. J. Physiol.: Cell Physiol. 2005, 288 (1), $\mathrm{C} 1-19$

(5) Sellers, L. A.; Allen, A.; Morris, E. R.; Ross-Murphy, S. B. Carbohydr. Res. 1988, 178, 93-110.

(6) Bansil, R.; Turner, B. S. Curr. Opin. Colloid Interface Sci. 2006, $11,164-170$

(7) Hong, Z.; Chasan, B.; Bansil, R.; Turner, B. S.; Bhaskar, K. R.; Afdhal, N. H. Biomacromolecules 2005, 6 (6), 3458-66.

(8) Bhaskar, K. R.; Gong, D. H.; Bansil, R.; Pajevic, S.; Hamilton, J. A.; Turner, B. S.; LaMont, J. T. Am. J. Physiol. 1991, 261 (5, Part 1), G827-32.

(9) Cao, X.; Bansil, R.; Bhaskar, K. R.; Turner, B. S.; LaMont, J. T.; Niu, N.; Afdhal, N. H. Biophys. J. 1999, 76 (3), 1250-8.

(10) Davis, S.; Inglis, A. Biorheology 1975, 12, 225-232.

(11) Sellers, L. A.; Allen, A. Symp. Soc. Exp. Biol. 1989, 43, 65-71.

(12) Sellers, L. A.; Allen, A.; Morris, E. R.; Ross-Murphy, S. B. Biorheology 1987, 24 (6), 615-23.

(13) Sellers, L. A.; Allen, A.; Morris, E. R.; Ross-Murphy, S. B. Biochim. Biophys. Acta 1991, 1115 (2), 174-9.

(14) Taylor, C.; Allen, A.; Dettmar, P. W.; Pearson, J. P. Biochim. Biophys. Acta 2004, 1674 (2), 131-8.

(15) Taylor, C.; Draget, K. I.; Pearson, J. P.; Smidsrod, O. Biomacromolecules 2005, 6 (3), 1524-30.

(16) Thim, L.; Madsen, F.; Poulsen, S. S. Eur. J. Clin. Invest. 2002, 32 (7), 519-27

(17) Celli, J.; Gregor, B.; Turner, B.; Afdhal, N. H.; Bansil, R.; Erramilli, S. Biomacromolecules 2005, 6 (3), 1329-33.

(18) Dawson, M.; Krauland, E.; Wirtz, D.; Hanes, J. Biotechnol. Prog. 2004, 20, (3), 851-7.

9) Suh, J.; Dawson, M.; Hanes, J. Adv. Drug Delivery Rev. 2005, 57475 (1), 63-78.

(20) Litt, M.; Khan, M. A.; Chakrin, L. W.; Wardell, J. R., Jr.; Christian, P. Biorheology 1974, 11 (2), 111-7.

(21) McCullagh, C M: Jamieson, A M: Blackwell, J; Gupta, R. Biopolymers 1995, 35 (2), 149-59. Invest. 1998, 58 (3), 259-64.

23) Tam, P. Y ; Katz, D. F. Berger, S. A Biorheology 1980, 17 (5-6) 465-78.

(24) Wolf, D. P.; Blasco, L.; Khan, M. A.; Litt, M. Fertil. Steril. 1977, 485 28 (1), 41-6. 
Biomacromolecules

PAGE EST: 6.4
Rheology of Gastric Mucin

(40) Muthukumar, M. Macromolecules 1989, 22 (12), 4656-4658. 516

(41) Stauffner, D.; Coniglio, A.; Adam, M. Adv. Polym. Sci. 1982, 44, 517 74.

(42) Hossain, K.; Miyanaga, K.; Maeda, H.; Nemoto, N. Biomacromol- 519 ecules 2001, 2 (2), 442-449.

(43) Baumgartner, H. K.; Montrose, M. H. Gastroenterology 2004, 126521 (3), 774-83.

(44) Barnes, H. J. Non-Newtonian Fluid Mech. 1999, 81 (1-2), 133- 523 178.

(45) Xu, J.; Tseng, Y.; Wirtz, D. J. Biol. Chem. 2000, 275 (46), 35886- 525 35892.

(46) Wilhelm, M. Macromol. Mater. Eng. 2002, 287 (2), 83-105. 527

(47) Denny, M. Nature 1980, 285, 160-161. 528

(48) Hyun, K.; Kim, S.; Ahn, K.; Lee, S. J. Non-Newtonian Fluid Mech. 529 2002, $107(1-3), 51-65$.

(49) Waigh, T.; Papagiannopoulos, A.; Voice, A.; Bansil, R.; Unwin, A.; 531 Dewhurst, C.; Turner, B.; Afdhal, N. Langmuir 2002, 18 (19), 7188- 532 7195.

(50) Deman, J.; Mareel, M.; Bruyneel, E. Biochim. Biophys. Acta 1973, 534 297 (2), 486-90.

51) Bansil, R · Celli, J ; Chasan, B ; Erramilli, S.; Hong, Z; Afdhal, N ; 59 Bhaskar, K. R.; Turner, B. pH-Dependent Gelation of Gastric Mucin. 537 In MRS 2005 Fall Proceedings, Symposium J; Langrana, J., Horkay, 538 F., Eds.; Materials Research Society: Warrendale, PA, 2005; Vol. 539 897.

(52) Bhaskar, K. R.; Garik, P.; Turner, B. S.; Bradley, J. D.; Bansil, R.; 541 Stanley, H. E.; LaMont, J. T. Nature 1992, 360 (6403), 458-61. 542

BM0609691 